\title{
Identification and molecular characterization of mutant line deficiency in three waxy proteins of common wheat (Triticum aestivum L.)
}

\section{Qian Liu}

Institute of Cereal and Oil Crops, HAAFS

Yaping Hu

Xingtai Medical College

Mengyun Hu

Institute of Cereal and Oil Crops, HAAFS

Lijing Sun

Institute of Cereal and Oil Crops, HAAFS

Xiyong Chen

Institute of Cereal and Oil Crops, HAAFS

Qianying Li

Institute of Cereal and Oil Crops, HAAFS

Peinan Wang

Institute of Cereal and Oil Crops, HAAFS

Li-an Wang

Hebei Normal University College of Life Sciences

Yingjun Zhang ( $\nabla$ zhangyingjun1977@163.com )

Institue of Cereal and Oil Crops, Hebei Academy of Agriculture and Forestry Sciences https://orcid.org/0000-0002-6476-980X

Hui Li

Institute of Cereal and Oil Crops, Hebei Academy of Agriculture and Forestry Sciences

\section{Research article}

Keywords: common wheat, granule bound starch synthase I, waxy wheat, amylose content, starch granules

Posted Date: December 3rd, 2019

DOI: https://doi.org/10.21203/rs.2.18059/v1 
License: (c) (i) This work is licensed under a Creative Commons Attribution 4.0 International License. Read Full License 


\section{Abstract}

Background: Starch is the main component of wheat (Triticum aestivum L.) grain and a key factor in determining wheat processing quality. The Wx gene encodes the granule bound starch synthase I (GBSS I) and is the sole gene responsible for amylose synthesis.

Results: A waxy mutant (Wx-null) was isolated by screening M3 seeds derived from 1.0\% EMS mutagenized materials with I2-KI staining of endosperm starch. SDS-PAGE electrophoresis confirmed that the Wx-null line lacked all three waxy proteins. DNA sequencing revealed three SNPs and a 3-bp InDel in the first exon, and a 16-bp InDel at the junction region of the first Wx-A1 intron from the Wx-null line. Six SNPs were identified in Wx-B1 gene of Wx-null line compared to the wild-type Gao 8901, including four missense mutations. One nonsense mutation was found at position 857 in the fourth exon, which resulted in a premature stop codon. Expression levels of Wx genes were dramatically reduced in the Wxnull line. Nonsense-mediated mRNA decay (NMD) may be triggered to degrade the non-functional Wx mRNA. There were no detectable differences in granule size and morphology between Wx-null and wildtype, but the $\mathrm{Wx}$-null line contained a larger proportion of B-type starch granules. The amylose content of the Wx-null line (0.22\%) was remarkably lower compared to the wild-type Gao 8901 (20.82\%). Total starch is also lower in the Wx-null line.

Conclusions: All three waxy proteins were non-functional in the Wx-null line. NMD may be the cause for reduced expression levels of Wx genes in the Wx-null line. The Wx-null line exhibited more B-type starch granules, dramatically lower amylose content, and decreased total starch. The Wx-null line may provide a potential waxy material with high agronomic performance in wheat breeding programs.

\section{Background}

Wheat (Triticum aestivum L.) is one of the most important crops worldwide. Starch is the main component of wheat grain and accounts for $65-75 \%$ of seed dry mass [1]. Wheat starch consists of two molecular types of glucose polymers: amylose and amylopectin. Amylose consists of long linear chains of D-glucopyranose units joined by a (1-4) glycosidic bonds, while amylopectin is highly branched and contains both a (1-4) and a (1-6) linkages of D-glucopyranose units [2]. Amylopectin molecules are significantly larger than amylose. Based on the content of amylose and amylopectin in starch, wheat is classified into non-waxy wheat starch, usually consisting of 18-29\% amylose [3], and waxy wheat starch, consisting mostly of amylopectin and less than $2 \%$ of amylose [4].

Various enzymes are involved in starch synthesis in the wheat endosperm, including ADPG pyrophosphorylase (AGPase; EC 2.7.7.27), starch branching enzyme (SBE; EC 2.4.1.18), starch debranching enzyme (DBE), granule bound starch synthase (GBSS; EC 2.4.1.21) and soluble starch synthase (SSS; EC 2.4.1.21) [5]. GBSS and SSS are the two forms of starch synthase (SS) [6]. It is generally known that in the endosperm amylose is primarily synthesized by GBSS I (the 'waxy' protein) [7] (Graybosch et al. 1998). Amylopectin synthesis is more complex, involving linked reactions catalyzed by 
enzymes SS (SSI, SSII, and SSIII), SBE and DBE. SBE plays an important role in amylopectin synthesis, which can introduce a-1,6-glucosidic linkages into a-polyglucans [8, 9]. In wild-type bread wheat, there are three GBSS I isoforms detected, one for each genome, encoded by orthologous $W x$ genes $(W x-A 1, W x-$ $B 1$, and $W x-D 1$ ) on chromosomes 7AS, 4AL (translocated from the original 7BS) and 7DS, respectively $[10,11]$. Amylose content in wheat starch is affected by GBSS I activity [12]. Wild-type wheat carries three functional $W x$ genes $(W x-A 1 a, W x-B 1 a$, and $W x-D 1 a) .$. When one or two $W x$ genes are non-functional, wheat often produces starch with reduced amylose content and is known as 'partial waxy' [13]. If all three $W x$ genes are null or non-functional, grains almost entirely consist of amylopectin and are termed 'waxy' $[7,10,14]$. $W x$ genes have additive but unequal effects on amylose content. For instance, $W x-B 1$ has the greatest effect on the amylose synthesis, followed by $W x-D 1$ and $W x-A 1[12,15]$.

Starch is a key factor determining the processing quality of wheat [16]. The two components of starch, amylose and amylopectin, differ significantly in their physicochemical properties. Amylose is primarily responsible for gel formation, especially regarding its structure and crystallinity [17], while amylopectin has an important role in retrogradation and long-term rheological characteristics of starch gels [18]. Amylose has large effects on starch gels initial firmness, while amylopectin is associated with firmness during storage [19]. Because gelatinized amylopectin absorbs more water than amylose, increased amylopectin can raise hot paste viscosity and swelling of starch-water suspensions of wheat flour [20]. As a result, changes in amylose or amylopectin content have profound impacts on end-use quality of wheat [21].

Waxy starch is composed predominantly of amylopectin, which gives waxy wheat its special processing characteristics. Compared with wild-type wheat starch, waxy wheat starch generally has higher peak viscosity and swelling volume, which can improve the quality of noodles including Japanese udon, Chinese-style ra-men and yellow alkaline noodles [22-24]. For example, as the proportion of waxy wheat starch increases from 0 to $52 \%$ in starch blends, peak viscosity increases from 210 to $640 \mathrm{BU}$ and peak temperature decreases from 95.5 to $70.0^{\circ} \mathrm{C}$. Salted noodles produced instead from blends of regular and waxy wheat flours are softer, more springy and cohesive [25] Additionally, waxy wheat starch shows a higher proportion of double-helical components and a lower proportion of single-helical components than non-waxy wheat starch [26]. Differential scanning calorimetry (DSC) and X-ray diffraction patterns showed that waxy wheat starch had higher crystallinity [27, 28]. Furthermore, waxy starch can influence bread-making quality, dough mixing characteristics and bread internal structure [28-30]. Water retention by waxy wheat starches is also much higher [25], increasing water absorption and loaf volume of bread [29-31]. Enhanced moisture-holding results in slowed staling and extension of bread shelf-life [32](Van Hung et al. 2007). Waxy wheat starch also has specific effects on gelatinization and retrogradation resistance [33]. Crumb structure of bread is partly controlled by starch gelatinization, and accordingly waxy starch has been shown to influence bread crumb structure, appearance and softness when added to normal wheat starch [34]. Starch retrogradation is also important to the industrial use of starch. Due to their slow retrogradation rates, waxy starches are preferred for chilled and frozen food products [35]. Waxy wheat starch has other important industrial applications including textiles, adhesives, papermaking, pharmaceuticals, ethanol and maltodextrins [7, 36-38]. 
Although waxy wheat flour alone is not suitable for food processing [25, 39], it is useful in salted noodles and bread blends by adjusting amylose content. Both the expansion rate and final product texture of extruded foods may be manipulated through control of amylose:amylopectin ratios. Waxy wheat provides millers with an efficient way to blend flours and achieve optimal amylose content levels [7]. The development and selection of waxy wheat are of importance in wheat breeding programs. Breeders worldwide have tried to develop their own waxy wheat varieties. In the present study, we report the isolation of Wx gene null lines of wild-type bread wheat cultivar Gao 8901 using EMS mutagenesis, and characterize their molecular mechanisms. Gao 8901 shows high bread-baking quality and is very popular in the Yellow and Huai winter wheat region of China. Due to its strong dough mixing properties, the $W x$ null line provides breeders valuable germplasms suitable for the production of bread and high-quality salted noodles. It may also be used to provide one or more waxy null alleles with high agronomic performance in wheat breeding programs.

\section{Results}

\section{Identification of the waxy protein null mutant line}

Seeds of common wheat cv. Gao 8901 were treated with a 1.0\% EMS solution, with a survival rate of M1 plants of $50-60 \%$. M1 plants were self-fertilized to produce the M2 generation. Half-seed staining using iodine potassium iodine solution $\left(0.1 \% \mathrm{I}_{2} / 1 \% \mathrm{KI}\right)$ was employed to screen waxy protein mutants. About 9,000 seeds from the M3 generation were screened. One seed showed brown-red color stained with $\mathrm{I}_{2}-\mathrm{KI}$ solution while others were dark blue (Fig. 1). We hypothesized that this seed was a waxy protein-deficient mutant and named it Wx-null.

\section{Waxy protein pattern analysis by SDS-PAGE electrophoresis}

The waxy protein pattern of the Wx-null line (M5 generation) was analyzed by SDS-PAGE electrophoresis, which showed that wild-type Gao 8901 had three waxy protein bands representing Wx-A1, Wx-B1, and WxD1, all of which were missing in the Wx-null mutant line (Fig. 2).

\section{Amylose content decreased significantly in Wx-null line}

Total starch and amylose content was determined for wild-type Gao 8901 and mutant line Wx-null (Table 1). Total starch content was $53.61 \%$ in Gao 8901 and $48.13 \%$ in Wx-null line. Furthermore, amylose content in Wx-null (0.22\%) was significantly lower than in the wild-type (20.82\%).

Table 1 Test of total starch and amylose content

\begin{tabular}{|c|c|c|}
\hline Materials & Total starch content (\%) & Amylose content (\%) \\
\hline Wild-type & $53.61 \pm 1.45^{*}$ & $20.82 \pm 0.08^{*}$ \\
\hline Wx-null & $48.13 \pm 1.73$ & $0.22 \pm 0.09$ \\
\hline
\end{tabular}


* Statistical significance was determined by a Student's $t$-test at $P<0.01$

\section{Starch granule characteristics of the Wx-null line}

Starch granule staining and scanning electron microscopy were employed to assess the effect of waxy protein deficiency on Wx-null line starch granule characteristics. Starch granules of wild-type Gao 8901 were dyed blue (Fig. 3A), while Wx-null line granules were stained red-brown (Fig. 3B). SEM observation of wild-type and Wx-null starch shapes revealed similar granule morphological features and size. A-type starch granules were $25 \mu \mathrm{m}$ in diameter and lenticular in shape, and B-type starch granules were $5 \mu \mathrm{m}$ in diameter and roughly spherical in both the wild-type and Wx-null line. However, the amount of B-type starch granules was much higher in Wx-null than in wild-type (Fig. 3).

\section{Wx gene sequences analysis of the Wx-null line}

Genomic DNA sequences of $W x$ gene of Gao 8901 were obtained. The gDNA of $W x$ gene from genomes $A, B$ and D were respectively 2,781 bp, 2,794 bp and 2,862 bp in total. They were named $W x-A 1$ (GenBank accession EU719608), $W x-B 1$ (GenBank accession EU719610) and $W x-D 1$ (GenBank accession EU719612), respectively. All $W x$ genes contained 11 exons and 10 introns. The gDNA sequences of $W x$ genes of the Wx-null line were also analyzed to detect mutant sites. DNA sequencing of the $W x-A 1$ gene from the Wx-null line revealed three SNPs and a 3-bp insertions and deletions (InDel) in the first exon. The G/C mutant at position 126 and A/T mutant at position 220 resulted in amino acid changes from methionine to isoleucine and serine to cystine, respectively. The 3-bp InDel (CAT) and the third SNP occurred at positions 315-317 and 320, respectively. Furthermore, a 16-bp InDel (GGCCGTAAGCTTGCGCCAC) was found at the junction region in the first intron (Fig. 4). The Wx-null $W x-$ $A 1$ gene was named $W x-A 1-n u l l(G e n B a n k$ accession EU719609). There was a total of six SNPs in the $W x-$ $B 1$ gene from the Wx-null line compared to wild-type Gao 8901, three of which were present in the first exon, one in the second, one in the third, and the last in the third intron (Fig. 5). The SNPs at positions 169 $(\mathrm{A} / \mathrm{G}), 244(\mathrm{~T} / \mathrm{C}), 430(\mathrm{G} / \mathrm{A})$ and $680(\mathrm{~T} / \mathrm{C})$ were missense mutations, which resulted in amino acid changes from lysine to glutamic acid, phenylalanine to leucine, glycine to serine, and leucine to proline, respectively. The SNP (C/T) at position 180 was a same-sense mutation. The $W x-B 1$ gene in the $W x$-null line was named Wx-B1-null (GenBank accession EU719611). One nonsense mutation (CAG to TAG) was found at position 857 in the fourth exon in Wx-null $W x-D 1$ gene line, which resulted in a premature stop codon (Fig. 6). The Wx-null Wx-D1 gene was then named Wx-D1-null (GenBank accession EU719613).

\section{Expression level of $W x$ gene decreases significantly in the Wx-null line}

RT-PCR was carried out to determine $W x$ gene expression level changes between wild-type Gao 8901 and Wx-null line. Total RNA was isolated from the seeds of Wx-null and wild-type at 15 DAF, reversetranscribed into cDNA and amplified by PCR. The Actin gene had the same PCR amplification level in 
both samples, indicating that their cDNA was at equal concentrations. The $W x$ genes were strongly expressed in seeds of wild-type Gao 8901. However, expression levels of $W x$ genes decreased significantly in the Wx-null line (Fig. 7).

\section{Discussion}

Waxy wheat has superior potential value for food production and non-food industries compared to nonwaxy wheat, but natural waxy wheat does not occur in nature. Identification of waxy protein-deficient lines play an important role in developing waxy wheat. Firstly, two cultivars with lower amylose content, Kanto 107 (K107) and K79, were found among Japanese wheat cultivars and shown to be $W x-A 1 / W x-B 1$ double null lines $[13,40]$. Later a worldwide sample screening of 1,960 wheat cultivars was performed, and one Chinese wheat (BaiHuo) was identified as null at the Wx-D1 locus [11]. By crossing K107 and BaiHuo, Nakamura [14] succeeded in producing the world's first completely waxy hexaploid wheat that lacked all isoforms of GBSS I. Since then, searches for novel $W x$ alleles in common wheat continued, with the goal of finding genetic variability to enable breeders to develop wheat lines with diverse starch properties and end-use quality characteristics. Yamamori [11] identified 177 cultivars with a null allele for the Wx-A1 protein, 159 cultivars carrying the null allele for the Wx-B1 protein, and nine Japanese cultivars lacking both the Wx-A1 and Wx-B1 proteins. Yamamori [11] also found variants of the waxy proteins WxA1c, Wx-B1c, and Wx-D1c. In recent years, many novel $W x$ alleles have been discovered with variable mutant frequencies. The fraction of null $W x-A 1$ is the highest (19 alleles), followed by $W x-B 1$ (17 alleles) and $W x-D 1$ (7 alleles) $[9,16,41-50]$. Additionally, the $W x-A 1$ null allele occurs frequently in Korean, Japanese and Turkish wheat, while wheat deficient in $W x-B 1$ is relatively frequent in Australia and India. The reason for the geographical distribution of null $W x$ alleles could be different origins of wheat germplasm across countries, or alternatively wheat breeders may have unconsciously selected for specific alleles due to the association between low amylose content and superior performance in noodle or specific bread production [7, 9]. However, no cultivars lacking both Wx-A1 and Wx-D1 proteins, both Wx$B 1$ and $W x-D 1$ proteins, or all three $W x$ proteins had bee detected $[11,51]$. Mutagenesis is a popular and effective way to create genetic variations in crops. Many $W x$ gene mutants have been isolated using EMS-induced assays50, 52-55]. In the present study, we isolated a Wx-null line lacking all three waxy proteins from a mutant population of Gao 8901 treated with EMS solution. To our knowledge, no other mutant line deficient in all three $W x$ genes $(W x-A 1, W x-B 1$, and $W x-D 1)$ has been identified.

Pre-mRNA alternative splicing (AS) plays an important role in gene expression diversity in eukaryotes. It is also estimated that a significant fraction of genes (about 20\%) undergo AS in plants [56,57]. InDel contributes to the generation of diversity in AS isoforms [58,59], by affecting splicing efficiency [60], the stability of the pre-mRNA structure, or the expression level of correctly spliced transcripts [61]. Through DNA sequencing, we identified three SNPs and a 3-bp InDel in the first exon, and a 16-bp InDel at the junction region in the first intron in the $W x-A 1$ gene of the Wx-null line (Fig. 4). The 16-bp InDel destroyed the GT-AT intron-exon boundary, which may have induced AS and loss of function of the $W x-A 1$ gene. The first reported null allele $W x-A 1 b$ (from K107) had a 23 bp deletion (in the second intron) also located at the exon-intron junction [62]. Additionally, Luo et al [50] reported an SNP (G to A) at the splicing site within 
the eighth intron, which caused incorrect RNA splicing and gene inactivation, suggesting a similar molecular mechanism for the above null $W x-A 1$ alleles. InDel is also a possible reason for non-functional $W x-A 1$ alleles. For example, a 173-bp insertion occurred in the fourth exon of null $W x-A 1$ (Turkey) [63], a 376-bp insertion in the $3^{\prime}$ untranslated region (UTR) resulted in aberrant mRNA of $W x-A 1 i$ [43], and a 738bp deletion affected the ninth to twelfth exons of $W x-A 10$ [9]. Among null $W x-B 1$ alleles, $W x-B 1 b$ (from $\mathrm{K} 107$ ) is the most common null type. There is a $67-\mathrm{kb}$ fragment deletion covering the entire coding region of the $W x-B 1$ gene $[12,62]$. InDel is also detected in several $W x-B 1$ null alleles, such as $W x-B 1 e, W x-B 1 k$, $W x-B 1 m$ and $W x-B 1 n[9,43,48]$. In the present study, sequence analysis revealed six SNPs in the $W x-B 1$ sequence of Wx-null line compared to wild-type. Four SNPs produced amino acid changes, and three located at a-helix or $\beta$-strand predicted by protein secondary structure (http://bioinf.cs.ucl.ac.uk). Therefore the amino acid changes may be the reason for the loss on function of the Wx-B1 protein. $W x-$ $D 1$ gene has the lowest variant frequency, and null $W x-D 1$ alleles are extremely rare in common wheat [64]. Previously researchers described a 588-bp fragment deletion in $W x$-D1b [62], a 724-bp fragment deletion in $W x-D 1 h[65]$ and a nucleotide substitution in $W x-D 1 g$ [66] among others. In this study, molecular characterization of $W x-D 1$ gene of $W x$-null line showed one nonsense mutation (from CAG to $T A G$ ) in the fourth exon resulting in a premature stop and non-function waxy protein. No similar mutation had been reported previously in $W x-D 1$. All three $W x$ genes exhibited novel variants in the $W x$-null line.

In this study, we also showed that $W x$ genes were strongly expressed in seeds of wild-type Gao 8901, but transcript levels were dramatically reduced in the Wx-null line, suggesting that post-transcriptional mechanisms controlling the quality of mRNA may inhibit the expression of non-function waxy proteins. In eukaryotes, a series of elaborate mechanisms ensure that gene expression is accurately executed and adapted to cellular needs. For example, mRNA surveillance assesses the quality of mRNA to ensure they are suitable for translation [67]. Truncated proteins encoded by mutant mRNA are potentially deleterious to the cell, and therefore nonsense-mediated mRNA decay (NMD) has evolved to recognize mRNAs containing premature termination codons (PTCs), prevent their synthesis and degrade them $[67,68]$. The decrease in expression level of $W x-D 1$ gene of the $W x$-null line may be due to NMD, caused by a premature termination codon (TAG) at the position 857 in the fourth exon, which reduced the length of the translation products from 604 to 189 amino acids. Evidence that premature termination codons can reduce mRNA level was first reported in studies of the Kunitz trypsin inhibitor (KTi) gene in soybean [69] and the phytohemag glutinin (PHA) gene in common bean [70]. NMD was also shown to affect the rice waxy gene [71] and $W x-A 1$ mutants [72]. When pre-mRNAs are spliced, mRNA acquires a multi-component complex at each exon-exon junction, which influences the subsequent steps of mRNA translation and is also a key effector of the fate of spliced mRNAs $[73,74]$. Additionally, the NMD pathway is not limited solely to mRNAs containing a PTC, but also works on aberrant transcripts with retained introns or extended 3' UTR owing to improper polyadenylation site usage [75-77]. In the present study, a 16-bp InDel at the exon-intron junction region in the first intron was found, which may lead to mistakes in premRNA splicing. A dramatic reduction in transcript level of $W x-A 1$ was observed, suggesting that mRNA was recognized and degraded by NMD. As for the $W x-B 1$ gene from the Wx-null line, only four missense mutants were found. The reason why the transcript level of $W x-B 1$ was so small remains unclear. $A$ 
possibility to be tested in future studies is whether there were mutant sites in the promoter region of $W x$ $B 1$ gene responsible for low expression.

GBSS I is the key enzyme for the biosynthesis of amylose in wheat, and its absence significantly hinders amylose synthesis. In the $W x$-null line all three $W x$ genes were non-functional, resulting in very low amylose content $(0.22 \%)$. Furthermore, the total starch content decreased in the Wx-null line $(48.13 \%)$ compared to wild-type Gao 8901 (53.61\%). Although GBSS I enzyme deficiency does not affect amylopectin synthesis, it causes a significant reduction in total starch biosynthesis [78]. Unlike corn starch granules characterized by more uniform size, wheat starch granules exhibit two main populations of large A-type and smaller B-type. As a result, waxy wheat starch shows different thermal and viscoelastic properties [79, 80], suggesting it may serve as a different substrate for starch cross-linking agents. Waxy wheat starch granules show an early developmental process. Starch granules begin to accumulate in the central endosperm cells of waxy wheat at $8 \mathrm{DAF}$, which is rarely observed in normal wheat. At $15 \mathrm{DAF}$, the number of starch granules in both waxy wheat and normal wheat increases, but Btype granules will only appear in waxy wheat. As a result, waxy wheat contains a larger proportion of Btype starch granules than normal wheat [26]. In the present study, we applied scanning electron microscopy to show that the amount of B-type starch granules in the Wx-null line was significantly higher than in wild-type, consistent with Yu's results [26]. However, the shape of A-type starch granules (25 $\mu \mathrm{m}$ in diameter and elliptical) did not differ between Wx-null line and wild-type Gao 8901, as previously reported by Jane et al [81]. A-type and B-type granules in wheat endosperm exhibit significant differences in functional properties and chemical composition [82]. Compared with A-type granules, B-type granules have higher gelatinization temperatures, lower transition enthalpy, and lower amylose content [83]. Starch granules features give waxy wheat special processing characteristics such as higher swelling power, higher peak viscosity, and gelatinization temperatures [26].

\section{Conclusions}

In the mutant line Wx-null, all three waxy proteins were non-functional. Novel $W x$ gene variants were found in Wx-null: three SNPs and a 3-bp InDel in the first exon, and a 16-bp InDel at the junction region of the first $W x-A 1$ intron; four missense mutations in $W x-B 1$ gene; one nonsense mutation at position 857 in $W x$-D1. Expression levels of $W x$ genes in the $W x$-null line were significantly reduced. The amylose content of the Wx-null line (0.22\%) was remarkably lower compared to the wild-type Gao 8901 (20.82\%). The Wxnull line contained a larger proportion of B-type starch granules. The Wx-null line may provide a potential waxy material with high agronomic performance in wheat breeding programs.

\section{Methods}

\section{Plant materials}

The Chinese common wheat cv. Gao 8901 was selected for developing EMS treated mutant lines. Gao 8901 released in 1998 has good baking-quality and is widely planted in the Huang and Huai winter wheat 
region. The seeds were originally obtained from the Institute of Agricultural Sciences of Gaocheng City, China. The Cereal Quality Testing Center of Hebei Province undertook the identification of the materials. Seed samples were stored at Wheat Research Center in the Institute of Cereal and Oil Crops, Hebei Academy of Agriculture and Forestry Sciences. Approximately 5000 dry seeds of Gao 8901 (M0) were soaked in distilled water for $12 \mathrm{~h}$ before being treated with $1.0 \% \mathrm{EMS}$ at room temperature $\left(25-27^{\circ} \mathrm{C}\right)$ for $6 \mathrm{~h}$ and then washed under running tap water for $4 \mathrm{~h}$ prior to seeding. In the subsequent growing season, treated seeds (M1) were individually sown and self-fertilized to produce the M2 generation. The M3 seeds were harvested from one spike per M2 plant and used for screening waxy protein mutants. Young leaves of Wx mutants (M4) were collected for DNA extraction and seeds were planted in a plant-to-row fashion to produce the M5 generation. The M5 seeds were used for RNA extraction, protein electrophoresis, amylose content determination, starch granule staining, and scanning electron microscopy analysis. All the experimental research and field studies were conducted in accordance with Chinese legislation.

\section{Half-seed screening of $W x$ mutant lines}

Waxy and non-waxy wheat seeds can be easily distinguished by staining. Starch from non-waxy wild-type containing amylose generates blue-black complexes with iodine, while starch from waxy protein mutants without amylose stains red-brown. M3 seeds were cut horizontally in two parts containing embryo and endosperm. The half seed containing the embryo was dipped into an iodine potassium iodine solution $\left(0.1 \% \mathrm{I}_{2} / 1 \% \mathrm{KI}\right)$ for $1 \mathrm{~min}$. On the basis of blue color intensity, seeds were grouped into low, intermediate, and high amylose categories for further analysis.

\section{Electrophoresis of waxy proteins}

Starch was extracted from M5 seeds following published methods [84]. Waxy proteins were separated using sodium dodecyl sulfate-polyacrylamide gel electrophoresis (SDS-PAGE) in a discontinuous buffer system ( $\mathrm{pH}: 6.8 / 7.8$ ). The polyacrylamide concentrations of stacking gel and resolving gel were respectively $4.5 \%$ and $15 \%$. Electrophoresis was performed at a constant voltage of $180 \mathrm{~V}$ for stacking gel and $220 \mathrm{~V}$ for resolving gel. Run time was approximately $4 \mathrm{~h}$ just after the tracking dye (bromophenol blue) had migrated off the gel. After electrophoresis, waxy proteins were detected by silver staining.

\section{Sequence analysis of $W x$ genes}

Genomic DNA was extracted from young leaves of $W x$ mutants (M4). Because the genomic sequences of $W x$ genes are about $2.8 \mathrm{~kb}$ and have high similarity at the $5^{\prime}$ and $3^{\prime}$ ends among $A, B$ and $D$ genomes, overlapping PCR was employed to amplify the complete $W x$ sequences. Primers were designed based on alignments of $W x$ genes sequences from the NCBI database (GenBank accession AB019622, AB019623, and AB019624) and primers reported by Slade and colleagues [85]. Primers were synthesized by Shanghai Sangon Biotech Co., Ltd. (http://www.sangon.com) and described in Table S1. PCR was 
performed in volumes of $20 \mu \mathrm{l}$, including $2 \mu \mathrm{l}$ of 10xEx Taq buffer, $0.5 \mu \mathrm{l}$ of dNTP ( $2.5 \mathrm{mM}$ of each dNTP), $1 \mu \mathrm{l}$ of each primer $(5 \mu \mathrm{M}), 1 \mathrm{U}$ of ExTaq and $80 \mathrm{ng}$ of template DNA. All reagents were from Takara Biotechnology Co., Ltd. (http://www.takara.com.cn). Reaction conditions were $94^{\circ} \mathrm{C}$ for $5 \mathrm{~min}$, followed by 8 cycles at $94^{\circ} \mathrm{C}$ for $30 \mathrm{~s}$, annealing at $70-65^{\circ} \mathrm{C}$ for $30 \mathrm{~s}\left(-1^{\circ} \mathrm{C}\right.$ per cycle $), 72^{\circ} \mathrm{C}$ for $1 \mathrm{~min}$, then 30 cycles at $94^{\circ} \mathrm{C}$ for $30 \mathrm{~s}$, annealing at $57-63^{\circ} \mathrm{C}$ for $30 \mathrm{~s}$ (depending on the primer), $72^{\circ} \mathrm{C}$ for $1 \mathrm{~min}$ and a final extension at $72^{\circ} \mathrm{C}$ for $10 \mathrm{~min}$. PCR products were separated by electrophoresis in $1 \%$ agarose gels stained with ethidium bromide and visualized using UV light. Targeted bands were recovered and cloned into the pEASY-T1 simple vector (Beijing TransGen Biotech Co., Ltd. http://www.transgen.com.cn) and sequenced by Shanghai Sangon Biotech Co., Ltd (http://www.sangon.com). Sequence analysis and characterization were performed using the software DNAMAN (http://www.lynnon.com) and Vector NTI Advance 10 (http://www.invitrogen.com). To guarantee sequence accuracy, PCR and DNA sequencing was repeated.

\section{Expression level analysis of $W x$ genes in mutant line using RT-PCR}

Total RNA was isolated from seeds of both $W x$ mutant line and wild-type Gao 8901 with three biological replicates at 15 days after flowering (DAF) using the Trizol method (www.tiangen.com). All samples were DNase-treated before reverse transcription. The first-strand CDNA was synthesized by MMLV reverse transcriptase (http://www.promega.com) using oligo (dT) as a primer. Reverse transcriptional products were adjusted to an equal concentration according to the PCR signal generated from the internal standard Actin house-keeping gene andused as templates for RT-PCR. All primers used in RT-PCR are listed in Table S1. RT-PCR was performed in total volumes of $20 \mu \mathrm{l}$, including $2 \mu \mathrm{l}$ of 10x ExTaq buffer, 0.5 $\mu \mathrm{l}$ of dNTP (2.5 mM of each dNTP), $1 \mu$ l of each primer $(5 \mu \mathrm{M}), 1 \mathrm{U}$ of ExTaq DNA polymerase and $80 \mathrm{ng}$ of template cDNA. Reaction condition was denaturation at $94^{\circ} \mathrm{C}$ for $3 \mathrm{~min}$, followed by 20 cycles of $94^{\circ} \mathrm{C}$ for $30 \mathrm{~s}, 59^{\circ} \mathrm{C}$ for $30 \mathrm{~s}$ and $72^{\circ} \mathrm{C}$ for $1 \mathrm{~min}$, and a final extension of $72^{\circ} \mathrm{C}$ for $5 \mathrm{~min}$. RT-PCR products were separated in $1 \%$ agarose gels, and bands were visualized with ethidium bromide.

\section{Amylose content analysis}

Total starch and amylose contents of mutant line and wild-type Gao 8901 were measured using the Total Starch Assay Kit and Amylose/Amylopectin Assay Kit (www.megazyme.com) according to manual instructions. All tests were performed on three replicates. Student's t-test assessed statistical differences between wild type and mutant line.

\section{Starch granule staining}

A small amount of starch granules was put onto a glass slide with a toothpick followed by a drop of dye solution $\left(50 \%(\mathrm{v} / \mathrm{v})\right.$ glycerol, $\left.0.1 \% \mathrm{I}_{2} / 1 \% \mathrm{KI}(\mathrm{w} / \mathrm{v})\right)$, then lightly covered with a cover slide and observed under a microscope. 


\section{Scanning electron microscopy analysis}

The granular morphology of starch was examined by scanning electron microscopy (SEM). Wheat seeds were transversely cut with a knife and fixed on circular aluminum stubs with double-sided sticky tape. Samples were sputter-coated with gold particles using an ionic sprayer (Eiko E-1020, Hitachi) and observed under a scanning electron microscope (S-570, Hitachi) with an accelerating voltage of $20 \mathrm{kV}$.

\section{Additional Files}

Additional file 1: Table S1 Primer sets used in this study. (DOXC, $15.6 \mathrm{~Kb}$ )

\section{Abbreviations}

GBSS I: Granule bound starch synthase l; EMS: Ethyl methanesulfonate; SDS-PAGE: Sodium dodecyl sulfate-polyacrylamide gel electrophoresis; PCR: Polymerase chain reaction; RT-PCR: Reverse transcription-polymerase chain reaction; SEM: Scanning electron microscopy; SNP: Single nucleotide polymorphism; InDel: Insertion and deletion; NMD: Nonsense-mediated mRNA decay; AGPase: ADPG pyrophosphorylase; SEB: Starch branching enzyme; DEB: Starch debranching enzyme; SSS: Soluble starch synthase; SS: Starch synthase; DSC: Differential scanning calorimetry; DAF: Days after flowering; NCBI: National Center for Biotechnology Information.

\section{Declarations}

\section{Ethics approval and consent to participate}

The experiments described in the manuscript were conducted according to ethical standards of China.

\section{Consent for publication}

Not applicable

\section{Availability of data and material}

All data generated or analyzed during this study are included in this published article and its supplemental data files.

\section{Competing interests}

The authors declare that they have no competing interests.

\section{Funding}

This work was supported by HAAFS Agriculture Science and Technology Innovation Project (4-08-03), National Key Research and Development Project (2018YFD0300503), Cultivation of New Wheat Varieties 
with High Yield, High Quality and Water-saving in North of Yellow and Huai Winter Wheat Region (2017YF00100603), Hebei Modern Agricultural Industry Technology System Innovation Team Construction (HBCT2018010201). The funders had no role in the design of the study, collection, analysis, or interpretation of the data, or writing of the manuscript.

\section{Authors' contributions}

QL and YH performed experiments and wrote the paper, $\mathrm{MH}$ performed the SDS-PAGE analysis, LS performed the RT-PCR analysis, XC perfomed the starch and amylose content analysis, QYL and PW performed gene sequencing, LW performed the scanning electron microscopy analysis, $\mathrm{YZ}$ and $\mathrm{HL}$ designed the experiments and assisted in writing the paper. All authors have read and approved the manuscript.

\section{Acknowledgments}

Not applicable

\section{Authors' Information}

${ }^{1}$ Institute of Cereal and Oil Crops, Hebei Academy of Agriculture and Forestry Sciences, Hebei Laboratory of Crop Genetics and Breeding, 162 Hengshan Street, Shijiazhuang 050035, China. ${ }^{2}$ Xingtai Medical College, 618 Gangtie North Road, Xingtai 054000, China. ${ }^{3}$ College of Life Sciences, Hebei Normal University, 20 Road East. 2nd Ring South, Shijiazhuang 050024, China.

\section{References}

1.Hurkman WJ, McCue KF, Altenbach SB, Korn A, Tanaka CK, Kothari KM, Johnson EL, Bechtel DB, Wilson JD, Anderson OD, DuPonta FM. Effect of temperature on expression of genes encoding enzymes for starch biosynthesis in developing wheat endosperm. Plant Sci.2003;164:873-81.

2.Huang XQ, Brûlé-Babel A. Development of genome-specific primers for homoeologous genes in allopolyploid species: the waxy and starch synthase //genes in allohexaploid wheat (Triticum aestivum L.) as examples. BMC Res Notes.2010;3:140.

3.Singh S, Singh N, Isono N, Noda T. Relationship of granule size distribution and amylopectin structure with pasting, thermal, and retrogradation properties in wheat starch. J Agric Food Chem.2009;58:1180-8.

4.Yasui T, Sasaki T, Matsuki J. Starch properties of a bread wheat (Triticum aestivum L.) mutant with an altered flour-pasting profile. J Cereal Sci.2002;35:11-6.

5.Emes M, Bowsher C, Hedley C, Burrell M, Scrase-Field E, Tetlow I. Starch synthesis and carbon partitioning in developing endosperm. J Exp Bot.2003;54:569-75. 
6.Jeon JS, Ryoo N, Hahn TR, Walia H, Nakamura Y. Starch biosynthesis in cereal endosperm. Plant Physiol Biochem.2010;48:383-92.

7.Graybosch RA. Waxy wheats: Origin, properties, and prospects. Trends Food Sci Technol.1998;9:13542.

8.Nakamura Y. Towards a better understanding of the metabolic system for amylopectin biosynthesis in plants: rice endosperm as a model tissue. Plant Cell Physiol.2002;43:718-25.

9.Guzmán C, Ortega R, Yamamori M, Peña RJ, Alvarez JB. Molecular characterization of two novel null waxy alleles in Mexican bread wheat landraces. J Cereal Sci.2015;62:8-14.

10.Nakamura T, Yamamori M, Hirano H, Hidaka S. Identification of three Wx proteins in wheat (Triticum aestivum L.). Biochem Genet.1993;31:75-86.

11.Yamamori M, Nakamura T, Endo T, Nagamine T. Waxy protein deficiency and chromosomal location of coding genes in common wheat. Theor Appl Genet.1994;89:179-84.

12.Saito $M$, Vrinten $P$, Ishikawa G, Graybosch R, Nakamura T. A novel codominant marker for selection of the null $W x-B 1$ allele in wheat breeding programs. Mol Breed.2009;23:209-17.

13. Nakamura T, Yamamori M, Hirano H, Hidaka S. Decrease of waxy $(\mathrm{Wx})$ protein in two common wheat cultivars with low amylose content. Plant Breed.1993;111:99-105.

14.Nakamura T, Yamamori M, Hirano H, Hidaka S, Nagamine T. Production of waxy (amylose-free) wheats. Mol Gen Genet.1995;248:253-9.

15.Miura $\mathrm{H}$, Sugawara A. Dosage effects of the three $W x$ genes on amylose synthesis in wheat endosperm. Theor Appl Genet.1996;93:1066-70.

16.Yamamori M, Quynh N. Differential effects of Wx-A1,-B1 and-D1 protein deficiencies on apparent amylose content and starch pasting properties in common wheat. Theor Appl Genet.2000;100:32-8.

17.Goodfellow B, Wilson R. A Fourier transform IR study of the gelation of amylose and amylopectin. Biopolymers.1990;30:1183-9.

18.Gudmundsson M. Retrogradation of starch and the role of its components. Thermochim Acta.1994;246:329-41.

19.Miles MJ, Morris VJ, Orford PD, Ring SG. The roles of amylose and amylopectin in the gelation and retrogradation of starch. Carbohydr Res.1985;135:271-81.

20.Martin JM, Talbert LE, Habernicht DK, Lanning SP, Sherman JD, Carlson G, Giroux MJ. Reduced amylose effects on bread and white salted noodle quality. Cereal Chem.2004;81:188-93. 
21.Klucinec JD, Thompson DB. Amylopectin nature and amylose-to-amylopectin ratio as influences on the behavior of gels of dispersed starch. Cereal Chem.2002;79:24-35.

22.Noda T, Tohnooka T, Taya S, Suda I. Relationship between physicochemical properties of starches and white salted noodle quality in Japanese wheat flours. Cereal Chem.2001;78:395-9.

23.Baik BK, Park C, Paszczynska B, Konzak C. Characteristics of noodles and bread prepared from double-null partial waxy wheat. Cereal Chem.2003;80:627-33.

24.Guo G, Jackson DS, Graybosch RA, Parkhurst AM. Asian salted noodle quality: Impact of amylose content adjustments using waxy wheat flour. Cereal Chem.2003;80:437-45.

25.Baik BK, Lee MR. Effects of starch amylose content of wheat on textural properties of white salted noodles. Cereal Chem.2003;80:304-9.

26.Yu X, Yu H, Zhang J, Shao S, Zhou L, Xiong F, Wang Z. Comparison of endosperm starch granule development and physicochemical properties of starches from waxy and non-waxy wheat. Int $\mathrm{J}$ Food Prop.2015;18:2409-21.

27.Shevkani K, Singh N, Bajaj R, Kaur A. Wheat starch production, structure, functionality and applications - a review. Int J Food Sci Technol.2017;52:38-58.

28.Zhang H, Zhang W, Xu C, Zhou X. Studies on the rheological and gelatinization characteristics of waxy wheat flour. Int J Biol Macromol.2014;64:123-9.

29.Blake LH, Jenner CF, Barber AR, Gibson RA, O'Neill BK, Nguyen QD. Effect of waxy flour blends on dough rheology and bread quality. Int J Food Sci Technol.2015;50:926-33.

30.Purna SKG, Miller RA, Seib PA, Graybosch RA, Shi YC. Volume, texture, and molecular mechanism behind the collapse of bread made with different levels of hard waxy wheat flours. J Cereal Sci.2011;54:37-43.

31.Ramachandran A, Hucl P, Briggs C. Functional characteristics of bread wheat (Triticum aestivum L.) near-isogenic lines differing at the waxy $(W x)$ locus. Cereal Chem.2016;93:77-85.

32.Bhattacharya M, Erazo-Castrejón SV, Doehlert DC, McMullen MS. Staling of bread as affected by waxy wheat flour blends. Cereal Chem.2002;79:178-82.

33.Sasaki T, Yasui T, Matsuki J. Effect of amylose content on gelatinization, retrogradation, and pasting properties of starches from waxy and nonwaxy wheat and their F1 seeds. Cereal Chem.2000;77:58-63.

34.Lee MR, Swanson BG, Baik BK. Influence of amylose content on properties of wheat starch and breadmaking quality of starch and gluten blends. Cereal Chem.2001;78:701-6. 
35. Hayakawa K, Tanaka K, Nakamura T, Endo S, Hoshino T. End use quality of waxy wheat flour in various grain-based foods. Cereal Chem.2004;81:666-72.

36.Jobling S. Improving starch for food and industrial applications. Curr Opin Plant Biol.2004;7:210-8.

37.Graybosch RA, Hansen LE. Functionality of chemically modified waxy, partial waxy and wild-type starches from common wheat. Starch-Stärke.2016;68:496-504.

38.Zhao R, Wu X, Seabourn B, Bean S, Guan L, Shi YC, Wilson J, Madl R, Wang D. Comparison of waxy vs. nonwaxy wheats in fuel ethanol fermentation. Cereal Chem.2009;86:145-56.

39.Morita N, Maeda T, Miyazaki M, Yamamori M, Miura H, Ohtsuka I. Dough and baking properties of high-amylose and waxy wheat flours. Cereal Chem.2002;79:491-5.

40.Kuroda A. A method of measuring amylose content and its variation in Japanese wheat cultivars and Kanto breeding lines (In Japanese). Japan J Breed.1989;39:142-3.

41.Yamamori M, Guzmán C. SNPs and an insertion sequence in five $W x-A 1$ alleles as factors for variant Wx-A1 protein in wheat. Euphytica.2013;192:325-38.

42.Ortega R, Guzmán C, Alvarez J. Molecular characterization of several Wx alleles in durum wheat. Biol Plant.2015;59:220-6.

43.Divashuk $M$, Klimushina $M$, Karlov $G$. Molecular genetic characteristics of the $W x-B 1 e$ allele from common wheat and applicability of the DNA markers for its identification. Russ J Genet.2011;47:142832.

44.Guzmán C, Caballero L, Álvarez JB. Molecular characterisation of the $W x-B 1$ allelic variants identified in cultivated emmer wheat and comparison with those of durum wheat. Mol Breed.2011;28:403-11.

45.Ayala M, Alvarez JB, Yamamori M, Guzmán C. Molecular characterization of waxy alleles in three subspecies of hexaploid wheat and identification of two novel $W x-B 1$ alleles. Theor Appl Genet.2015;128:2427-35.

46.Yamamori M. Amylose content and starch properties generated by five variant $W x$ alleles for granulebound starch synthase in common wheat (Triticum aestivum L.). Euphytica.2009;165:607-14.

47.Yamamori M, Yamamoto K. Effects of two novel $W x-A 1$ alleles of common wheat (Triticum aestivum L.) on amylose and starch properties. J Cereal Sci.2011;54:229-35.

48.Zhang LL, Chen H, Luo M, Zhang XW, Deng M, Ma J, Qi PF, Wang JR, Chen GY, Liu YX, Pu ZE, Li W, Lan $X J$, Wei YM, Zheng YL, Jiang QT. Transposon insertion resulted in the silencing of $W x-B 1 n$ in Chinese wheat landraces. Theor Appl Genet.2017;130:1321-30. 
49.Rodriguez-Quijano M, Nieto-Taladriz MT, Carrillo JM. Polymorphism of waxy proteins in Iberian hexaploid wheats. Plant Breed.1998;117:341-4.

50.Luo M, Ding J, Li Y, Tang H, Qi P, Ma J, Wang J, Chen G, Pu Z, Li W, Li Z, Harwood W, Lan X, Deng M, Lu $Z$, Wei $Y$, Zheng $Y$, Jiang Q. A single-base change at a splice site in $W x-A 1$ caused incorrect RNA splicing and gene inactivation in a wheat EMS mutant line. Theor Appl Genet.2019;132:2097-109.

51.Graybosch R, Peterson C, Hansen L, Rahman S, Hill A, Skerritt J. Identification and characterization of US wheats carrying null alleles at the $w x$ loci. Cereal Chem.1998;75:162-5.

52.Kiribuchi-Otobe C, Yanagisawa T, Yamaguchi I, Yoshida H. Wheat mutant with waxy starch showing stable hot paste viscosity. Cereal Chem.1998;75:671-2.

53.Yasui T. Waxy and low-amylose mutants of bread wheat (Triticum aestivum L.) and their starch, flour and grain properties. JARQ.2006;40:327-31.

54.Yasui T, Ashida K, Sasaki T. Chain-length distribution profiles of amylopectin isolated from endosperm starch of waxy and low-amylose bread wheat (Triticum aestivum L.) lines with common genetic background. Starch-Stärke.2009;61:677-86.

55.Yanagisawa T, Kiribuchi-Otobe $\mathrm{C}$, Yoshida H. An alanine to threonine change in the Wx-D1 protein reduces GBSS I activity in waxy mutant wheat. Euphytica.2001;121:209-14.

56.Wang BB, Brendel V. Genomewide comparative analysis of alternative splicing in plants. Proc Natl Acad Sci.2006;103:7175-80.

57.Barbazuk WB, Fu Y, McGinnis KM. Genome-wide analyses of alternative splicing in plants: opportunities and challenges. Genome Res.2008;18:1381-92.

58.Huang X, Lu G, Zhao Q, Liu X, Han B. Genome-wide analysis of transposon insertion polymorphisms reveals intraspecific variation in cultivated rice. Plant Physiol.2008;148:25-40.

59.Warnefors M, Pereira V, Eyre-Walker A. Transposable elements: insertion pattern and impact on gene expression evolution in hominids. Mol Biol Evol.2010;27:1955-62.

60.Wilkinson $\mathrm{M}$, Lenton J, Holdsworth $\mathrm{M}$. Transcripts of $V p-1$ homoeologues are alternatively spliced within the Triticeae tribe. Euphytica.2005;143:243-6.

61.Yang Y, Zhao X, Xia L, Chen X, Xia X, Yu Z, He Z, Röder M. Development and validation of a Viviparous-1 STS marker for pre-harvest sprouting tolerance in Chinese wheats. Theor Appl Genet.2007;115:971-80.

62.Vrinten P, Nakamura T, Yamamori M. Molecular characterization of waxy mutations in wheat. Mol Gen Genet.1999;261:463-71. 
63.Saito M, Konda M, Vrinten P, Nakamura K, Nakamura T. Molecular comparison of waxy null alleles in common wheat and identification of a unique null allele. Theor Appl Genet.2004;108:1205-11.

64.Van Hung P, Maeda T, Morita N. Waxy and high-amylose wheat starches and flours - characteristics, functionality and application. Trends Food Sci Technol.2006;17:448-56.

65.Monari AM, Simeone MC, Urbano M, Margiotta B, Lafiandra D. Molecular characterization of new waxy mutants identified in bread and durum wheat. Theor Appl Genet.2005;110:1481-9.

66.Yamamori $\mathrm{M}$, Yasui T. Combination of null, variant, and mutant $W x$ alleles in common wheat leads to amylose variations ranging from waxy to normal. Crop Sci.2016;56:644-53.

67.Wagner E, Lykke-Andersen J. mRNA surveillance: the perfect persist. J Cell Sci.2002;115:3033-8.

68.Wilusz CJ, Wang W, Peltz SW. Curbing the nonsense: the activation and regulation of mRNA surveillance. Genes Dev.2001;15:2781-5.

69.Jofuku KD, Schipper RD, Goldberg RB. A frameshift mutation prevents Kunitz trypsin inhibitor mRNA accumulation in soybean embryos. Plant Cell.1989;1:427-35.

70.Voelker TA, Moreno J, Chrispeels MJ. Expression analysis of a pseudogene in transgenic tobacco: a frameshift mutation prevents mRNA accumulation. Plant Cell.1990;2:255-61.

71.Isshiki M, Yamamoto Y, Satoh H, Shimamoto K. Nonsense-mediated decay of mutant waxy mRNA in rice. Plant Physiol.2001;125:1388-95.

72.Saito $M$, Nakamura $T$. Two point mutations identified in emmer wheat generate null $W x-A 1$ alleles. Theor Appl Genet.2005;110:276-82.

73.Maquat LE. Nonsense-mediated mRNA decay: splicing, translation and mRNP dynamics. Nat Rev Mol Cell Biol.2004;5:89-99.

74.Schell T, Kulozik AE, Hentze MW. Integration of splicing, transport and translation to achieve mRNA quality control by the nonsense-mediated decay pathway. Genome Biol.2002;3:reviews1006.1-6.

75.Mitrovich QM, Anderson P. Unproductively spliced ribosomal protein mRNAs are natural targets of mRNA surveillance in C. elegans. Genes Dev.2000;14:2173-84.

76.Ruiz-Echevarría MJ, Peltz SW. The RNA binding protein Pub1 modulates the stability of transcripts containing upstream open reading frames. Cell.2000;101:741-51.

77.Muhlrad D, Parker R. Aberrant mRNAs with extended 3' UTRs are substrates for rapid degradation by mRNA surveillance. RNA.1999;5:1299-307. 
78.Chen GX, Zhou JW, Liu YL, Lu XB, Han CX, Zhang WY, Xu YH, Yan YM. Biosynthesis and regulation of wheat amylose and amylopectin from proteomic and phosphoproteomic characterization of granulebinding proteins. Sci Rep.2016;6:33111.

79.Yasui T, Matsuki J, Sasaki T, Yamamori M. Amylose and lipid contents, amylopectin structure, and gelatinisation properties of waxy wheat (Triticum aestivum) starch. J Cereal Sci.1996;24:131-7.

80. Hayakawa K, Tanaka K, Nakamura T, Endo S, Hoshino T. Quality characteristics of waxy hexaploid wheat (Triticum aestivum L.): Properties of starch gelatinization and retrogradation. Cereal Chem.1997;74:576-80.

81.Jane JL, Kasemsuwan T, Leas S, Zobel H, Robyt JF. Anthology of starch granule morphology by scanning electron microscopy. Starch-Stärke.1994;46:121-9.

82.Kim HS, Huber KC. Physicochemical properties and amylopectin fine structures of A-and B-type granules of waxy and normal soft wheat starch. J Cereal Sci.2010;51:256-64.

83.Van Hung P, Morita N. Physicochemical properties of hydroxypropylated and cross-linked starches from A-type and B-type wheat starch granules. Carbohydr Polym.2005;59:239-46.

84.Yamamori M, Endo T. Variation of starch granule proteins and chromosome mapping of their coding genes in common wheat. Theor Appl Genet.1996;93:275-81.

85.Slade AJ, Fuerstenberg SI, Loeffler D, Steine MN, Facciotti D. A reverse genetic, nontransgenic approach to wheat crop improvement by TILLING. Nat Biotechnol.2005;23:75-81.

\section{Figures}




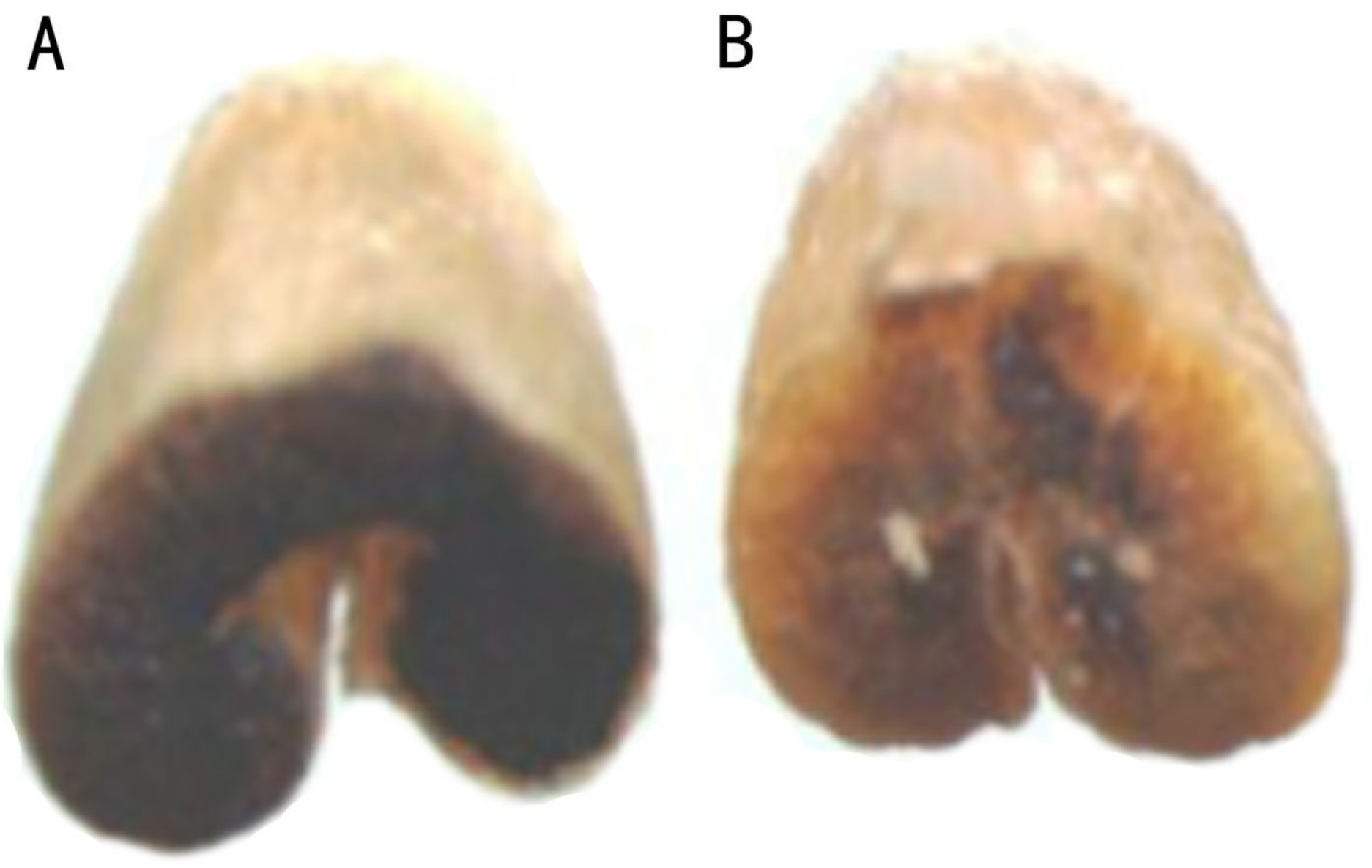

\section{Figure 1}

Identification of waxy protein-deficient mutant line stained with I2-KI solution. a: wild-type; b: Wx-null line.
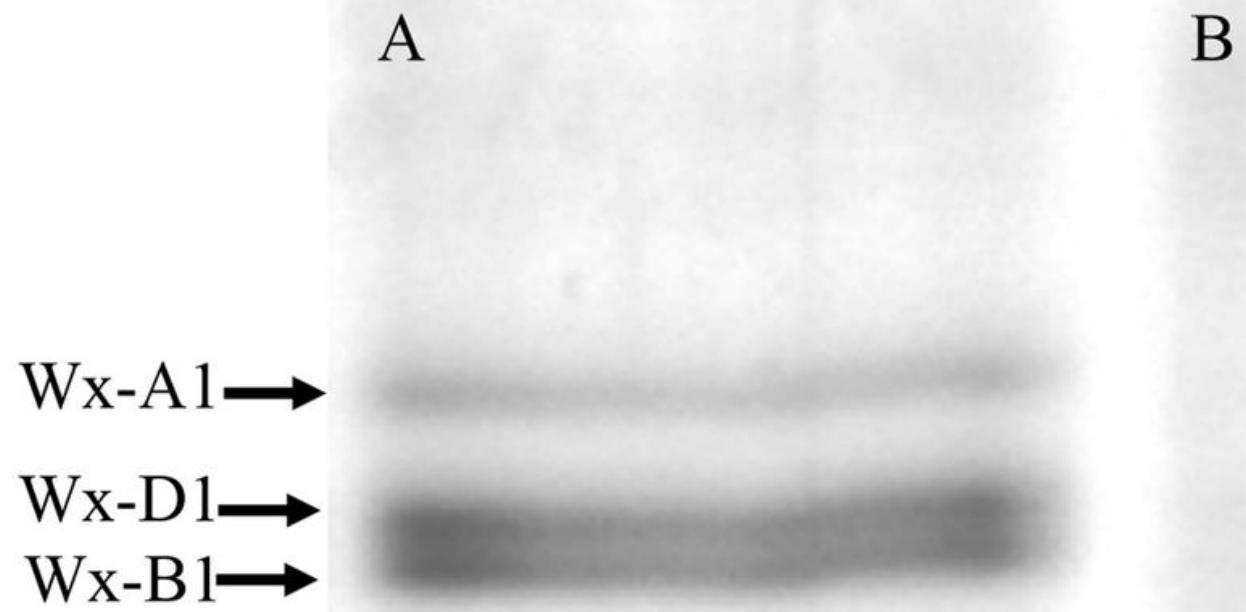
Figure 2

SDS-PAGE analysis of the waxy protein mutant line. a: wild-type; b: Wx-null line.

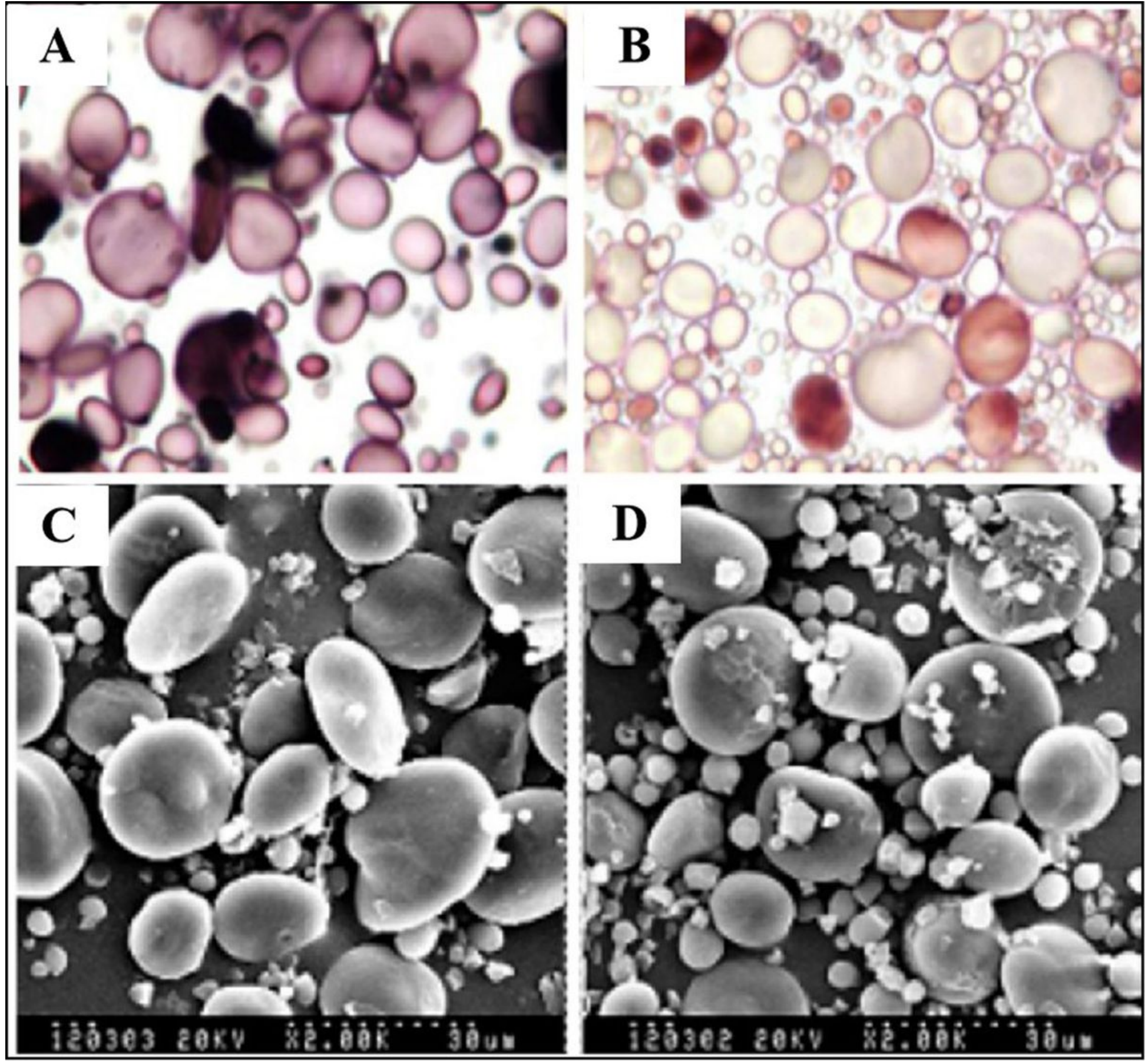

Figure 3

Starch granule staining and structure analysis. Starch granule staining by I2-KI solution (1000 diameters). a: wild-type; b: Wx-null line. Starch structure visualized by SEM (2000 diameters). c: wild-type; d: Wx-null line. 


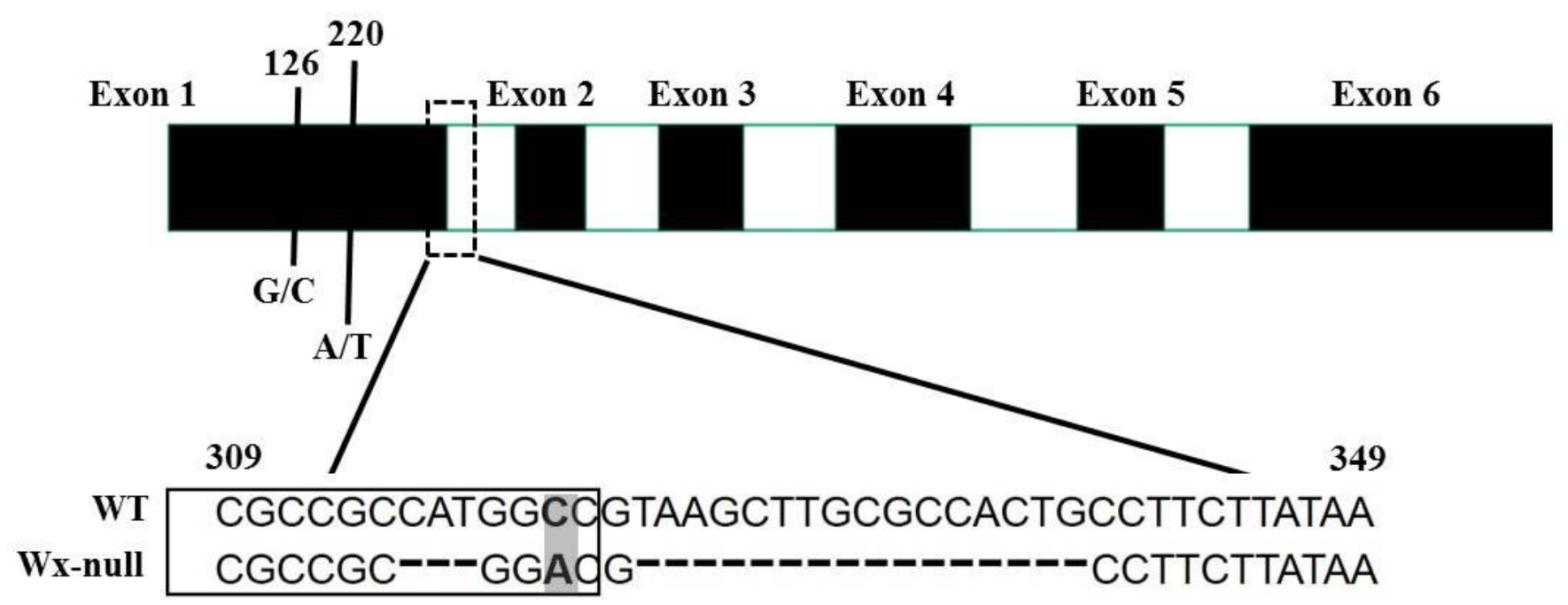

Figure 4

Analysis of Wx-A1 gene sequences in wild-type and Wx-null line. Black and white boxes respectively indicate exons and introns. Numbers show base position. DNA sequences at the border of the first exon are boxed. SNP is indicated by the shaded area. Dashed line indicates the InDel.

\section{Exon 2 Exon 3}

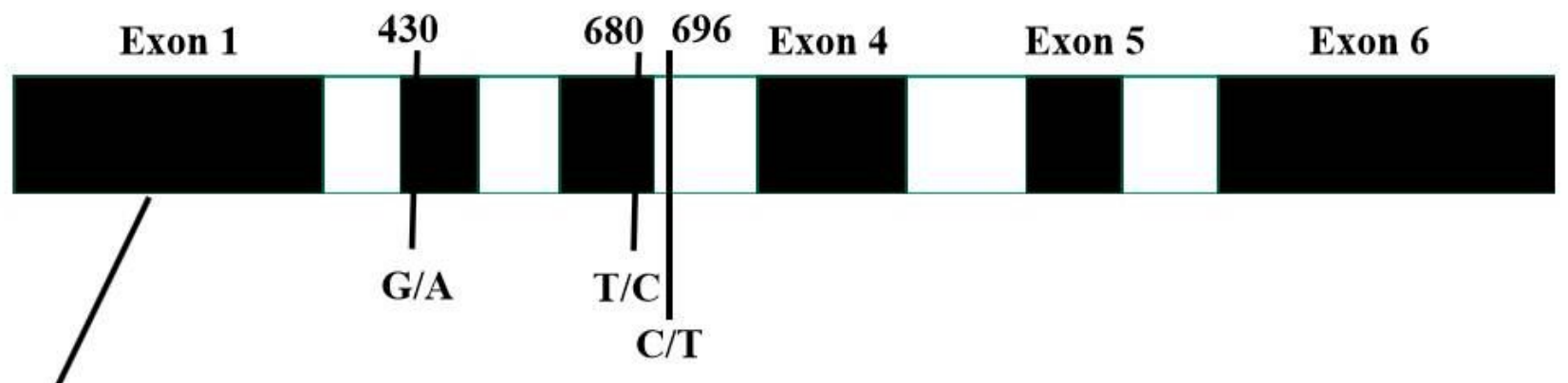

169

180

WT CAAAGCCGGAAAGCGCACCGCGGGACCCGGCGGTGCCTCTCCATGGTG Wx-null CAAAGCCGGGAAGCGCACCGTGGGACCCGGCGGTGCCTCTCCATGGTG 244

WT GTGCGCGCCACGGGCAGCGCCGGCATGAACCTCGTGTTCGTCGGCGCC wx-null GTGCGCGCCACGGGCAGCGCCGGCATGAACCTCGTGCTCGTCGGCGCC

Figure 5

Analysis of Wx-B1 gene sequences in wild-type and Wx-null line. Black and white boxes respectively indicate exons and introns. Numbers show base position. SNPs is indicated by the shaded area. 


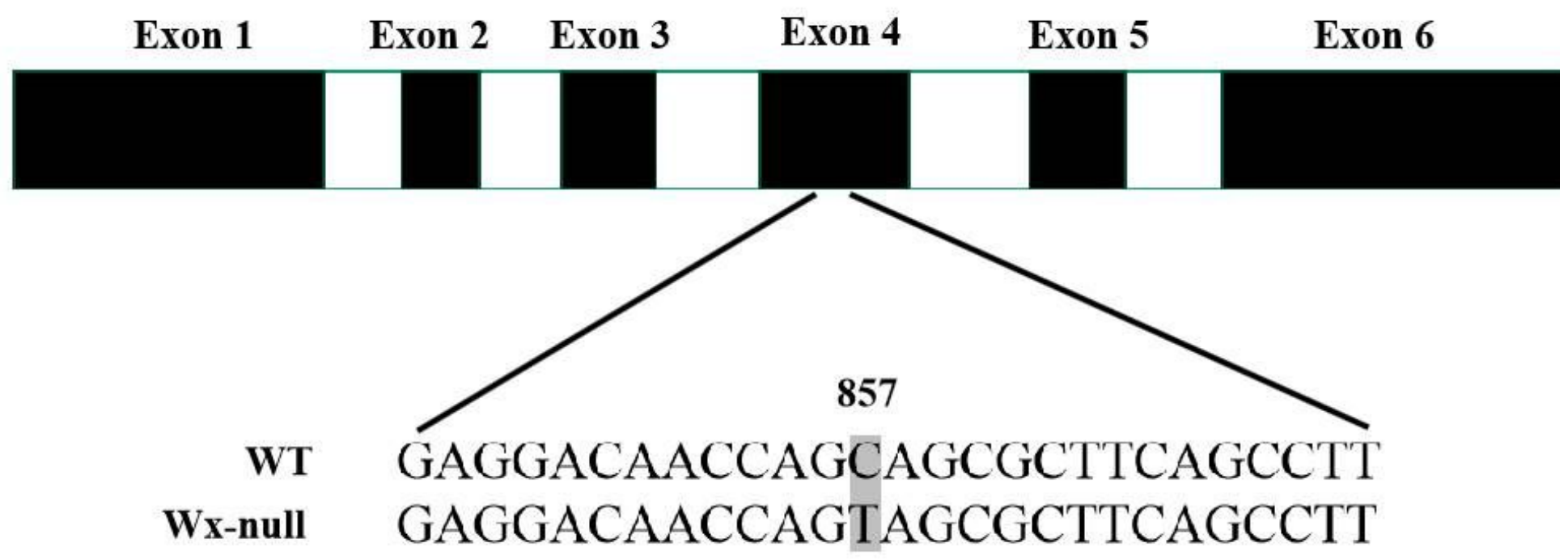

Figure 6

Analysis of Wx-D1 gene sequences in wild-type and Wx-null line. Black and white boxes respectively indicate exons and introns. Numbers show base position. SNP is indicated by the shaded area.

\section{WT Wx-null}

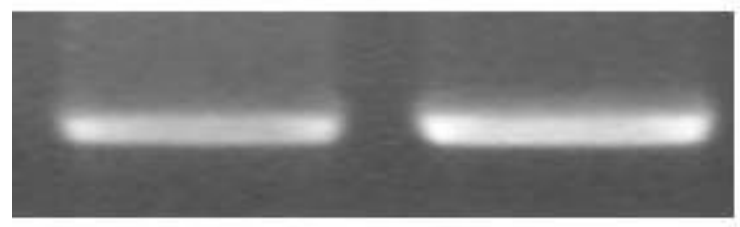

Actin

\section{$W x$}

Figure 7

Expression analysis of Wx gene using RT-PCR. Gene names shown on the right side.

\section{Supplementary Files}

This is a list of supplementary files associated with this preprint. Click to download.

- TableS1Primersetsusedinthisstudy.docx 